\title{
Association of Lipid Accumulation Product with Gestational Diabetes Mellitus: A Hospital Based Study
}

\author{
Shaanthy Gunasingh T K ${ }^{1}$, Padmalatha $\mathbf{D}^{1 *}$, Indhumathi Nachiyar ${ }^{1}$, Prasanna Karthik Suthakaran² and Kannan $\mathbf{R}^{2}$ \\ ${ }^{1}$ Department of Obstetrics and Gynaecology, Government Kilpauk Medical College Hospital, Kilpauk, Chennai, Tamilnadu, India \\ ${ }^{2}$ Department of General Medicine, Saveetha Medical College Hospital, Saveetha University, Thandalam, Chennai, Tamilnadu, India
}

\section{ABSTRACT}

Background: Gestational Diabetes Mellitus (GDM) is an emerging problem which affects a large number of pregnant women in India. Women with GDM have been shown to have abnormal lipid profiles with higher serum triacylglycerol concentrations but lower LDL levels. Early detection reduces adverse maternal and foetal outcome. The Lipid Accumulation Product (LAP) is an index of fat distribution and assessment which can be easily measured in an outpatient setup.

Methods: This study was a hospital based case control study. Cases were 30 pregnant women, newly diagnosed with GDM in their 2 nd trimester while controls were 30 apparently healthy pregnant women without risk factors for GDM. Lipid accumulation product (LAP) was computed by multiplying a sex-specific estimate of waist circumference and the fasting triglyceride concentration, $\mathrm{LAP}=(\mathrm{WC}$ [cm] -58$) \mathrm{x}$ TGL $[\mathrm{mmol} / \mathrm{L}]$.

Result: The mean age of patients with GDM was higher when compared to controls. $(28.17 \pm 3.34$ vs $24.40 \pm 3.07$, p $<0.0001)$ The lipid profile showed a significantly higher value of serum triglycerides among cases while the differences in HDL were not statistically significant. Lipid accumulation product (LAP) in GDM patients was found to be significantly elevated when compared to controls. (133.43 \pm 64.02 vs $62.89 \pm 30.68, \mathrm{p}<0.0001)$

Conclusion: Calculation of LAP can be done to identify the degree of risk for developing GDM. So LAP can possibly serve as in future as a screening tool for the diagnosis of GDM in an outpatient setting in resource poor settings.

Keywords: LAP, Gestational Diabetes Mellitus, Gdm, Fat Distribution

\section{Introduction}

Gestational Diabetes Mellitus (GDM) is defined as carbohydrate intolerance of variable severity, with onset or first recognition during pregnancy.(1,2) The incidence of GDM has increased over the past few decades in parallel with the increase in rates of obesity and type 2 diabetes mellitus, and this trend is expected to continue in the near future too.(3)

Physiological hyperlipidemia, is associated with an increase in TGL, LDL and VLDL levels and a decrease in HDL - C levels. Women with GDM have also been shown to have higher serum triacylglycerol concentrations but lower LDL than normal pregnant women. The inverse association between high-triglyceride and low HDL Cholesterol pattern is readily apparent in women with GDM.(4) This ratio (TG/HDL-C) has been confirmed as a clinical indicator of insulin resistance.(5) Higher plasma insulin levels may suppress fatty acid oxidation and thereby contribute to the elevated triglycerides in blood. Hyperinsulinemia is also known to enhance hepatic very low-density lipoprotein (VLDL) synthesis, which may further contribute to increased plasma triglycerides and
LDL - C levels. Resistance to the action of insulin on lipoprotein lipase in peripheral tissues can also contribute to the elevated triglyceride and $\mathrm{LDL}-\mathrm{C}$ levels.

A study reported that the "hypertriglyceridemic waist" phenotype identified in the first trimester could be a simple early screening tool for gestational glucose intolerance. (6) The hypertriglyceridemic waist is a clinical marker of visceral obesity that is defined as the simultaneous presence of abdominal obesity and hypertriglyceridemia. (7) Enquobahrie et al found that women with plasma triacylglycerol levels $\geq 137 \mathrm{mg} / \mathrm{dl}$ displayed a 3.5 fold increased risk of GDM, and each $20 \mathrm{mg} / \mathrm{dL}$ increase in triacylglycerol levels led to a $10 \%$ increase in GDM risk; however, no other associations could be observed between lipid changes and GDM risk in that study(8).

Lipid accumulation is defined and measured specifically in those contexts where accumulation may represent a physiologic danger. $(9,10)$ These contexts might be described as lipid over accumulation.(11) To simplify the recognition of lipid over accumulation, researchers have devised empirical formulae which can serve as risk 
markers. This study was conducted in an attempt to find the association of lipid accumulation product with GDM.

\section{Materials and Methods}

This study was a hospital based case control study among the pregnant women who attended the outpatient and inpatient services of a tertiary care referral hospital in South India. Before the start of the study, clearance was obtained from both the institutional Scientific Research Board (SRB) and the Institutional Ethics Committee (IEC). Written informed consent was obtained from eligible participants in the native language prior to the commencement of the study.

The study population was determined to be two groups of 30 participants each, cases were 30 pregnant women newly diagnosed with GDM in their 2nd trimester while controls were 30 apparently healthy pregnant women in their 2nd trimester without risk factors for GDM. Cases were excluded if there were multiple gestations. Data collection was done using a semi structured proforma.

All anthropometric measurements were done using a plastic measuring tape by the same investigator. BMI was calculated after measuring the height in metres and weight in kilograms. The waist circumference was measured using measuring tape at the level of umbilicus, at the end of quiet inspiration with both feet touching the ground and arms hanging freely. A simple index for estimating lipid accumulation in adults, Lipid Accumulation Product (LAP), was described by Kahn.(12) It was based on a combination of two measurements; waist circumference (WC) - a measure of truncal fat that includes the visceral depot and fasting concentration of serum triglycerides (TGL). LAP was computed by multiplying a sex- specific estimate of waist circumference and the fasting triglyceride concentration; in women, LAP $=(\mathrm{WC}[\mathrm{cm}]$ - 58) x TGL [mmol/L]

A 10 - 12 hour fasting sample was collected for estimation of the lipid profile. The data was analysed using SPSS for Windows 24 package with appropriate parametric and nonparametric methods.

\section{Result}

The baseline characteristics of the study population are described in Table 1. The mean age of patients with GDM was higher when compared to controls and the difference was statistically significant. $(28.17 \pm 3.34$ vs $24.40 \pm 3.07$, $\mathrm{p}<0.0001)$ The mean gestational age of cases and controls were comparable at baseline. The distribution of age and gestational age of cases and controls is as shown. (Figures 1 and 2)

The mean heights of the women were not different between the two groups but their weights were significantly different. The mean Body Mass Index (BMI) and waist circumference (WC) was $23.59 \pm 4.19$ and $88.46 \pm 7.10$ respectively among controls and $29.85 \pm 4.52$ and 102.12 \pm 6.96 respectively among GDM patients and these differences were highly significant ( $p$ value $<0.0001$ ). The distribution of BMI among cases and controls is shown in Figure 3. The lipid profile showed significantly higher values of triglycerides among cases while the difference in HDL - Cholesterol levels were not statistically significant. Linear correlation analysis revealed a moderately strong linear relationship between OGCT and LAP. (LAP = $0.6978 *$ OGCT $+0.8578, \mathrm{r}=0.6446, \mathrm{r}^{2}=0.4155, \mathrm{P}<$ 0.00001). (Figure 4)

Table 1: Baseline Characteristics and LAP of the cases and controls.

\begin{tabular}{|l|c|c|c|}
\hline & CASES & CONTROL & P value \\
\hline Age(in yrs) & $26.18 \pm 3.34$ & $23.96 \pm 3.07$ & $<0.0001$ \\
\hline Gestational Age(in weeks) & $21.71 \pm 3.13$ & $20.27 \pm 2.53$ & 0.4657 \\
\hline GCT(in mg/dL) & $172.65 \pm 32.17$ & $93.50 \pm 11.42$ & $<0.0001$ \\
\hline Height(in cm) & $154.19 \pm 4.32$ & $152.40 \pm 5.19$ & 0.9806 \\
\hline Weight(in kg) & $71.26 \pm 9.62$ & $54.42 \pm 9.85$ & $<0.0001$ \\
\hline BMI(in kg/m2) & $28.81 \pm 4.11$ & $23.14 \pm 5.19$ & $<0.0001$ \\
\hline Waist Circumference(in cm) & $106.12 \pm 7.16$ & $82.49 \pm 6.67$ & $<0.0001$ \\
\hline TGL(in mmol/L) & $2.49 \pm 1.07$ & $1.84 \pm 0.69$ & 0.0002 \\
\hline HDL(in mmol/L) & $0.73 \pm 0.30$ & $0.78 \pm 0.21$ & 0.3499 \\
\hline LAP & $133.43 \pm 64.02$ & $62.89 \pm 30.68$ & $<0.0001$ \\
\hline
\end{tabular}




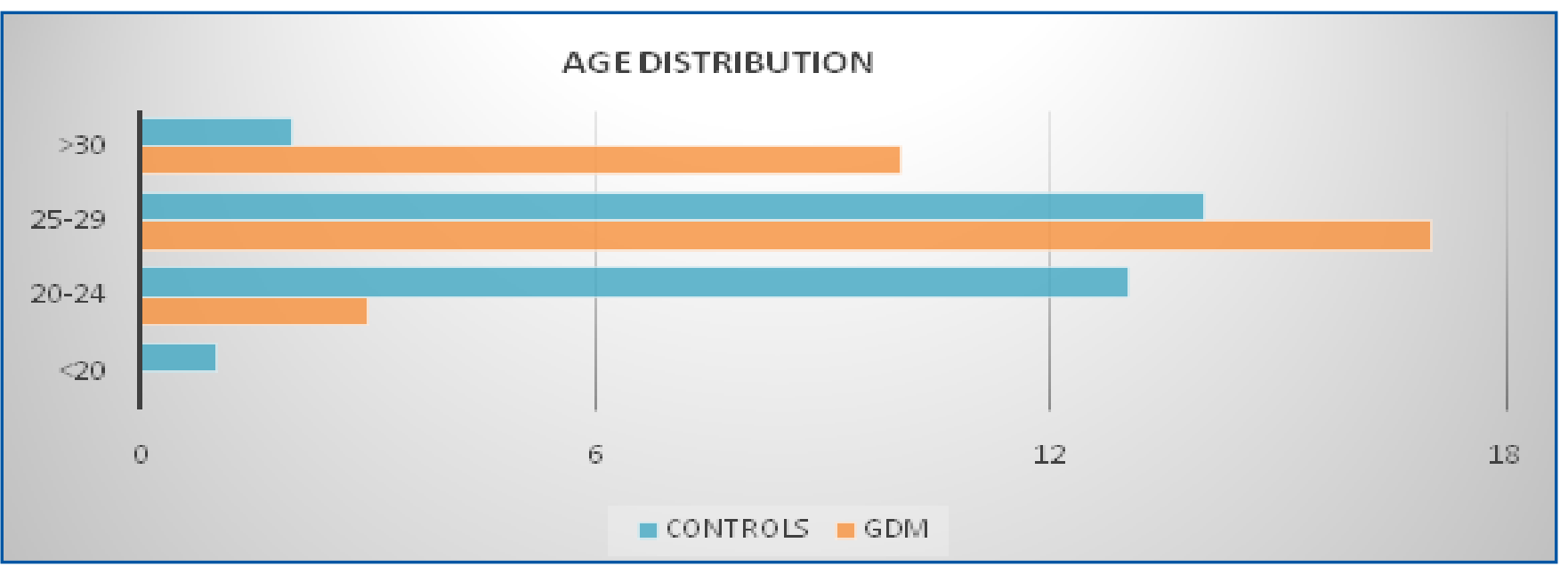

Fig. 1: Age distribution among cases and controls.

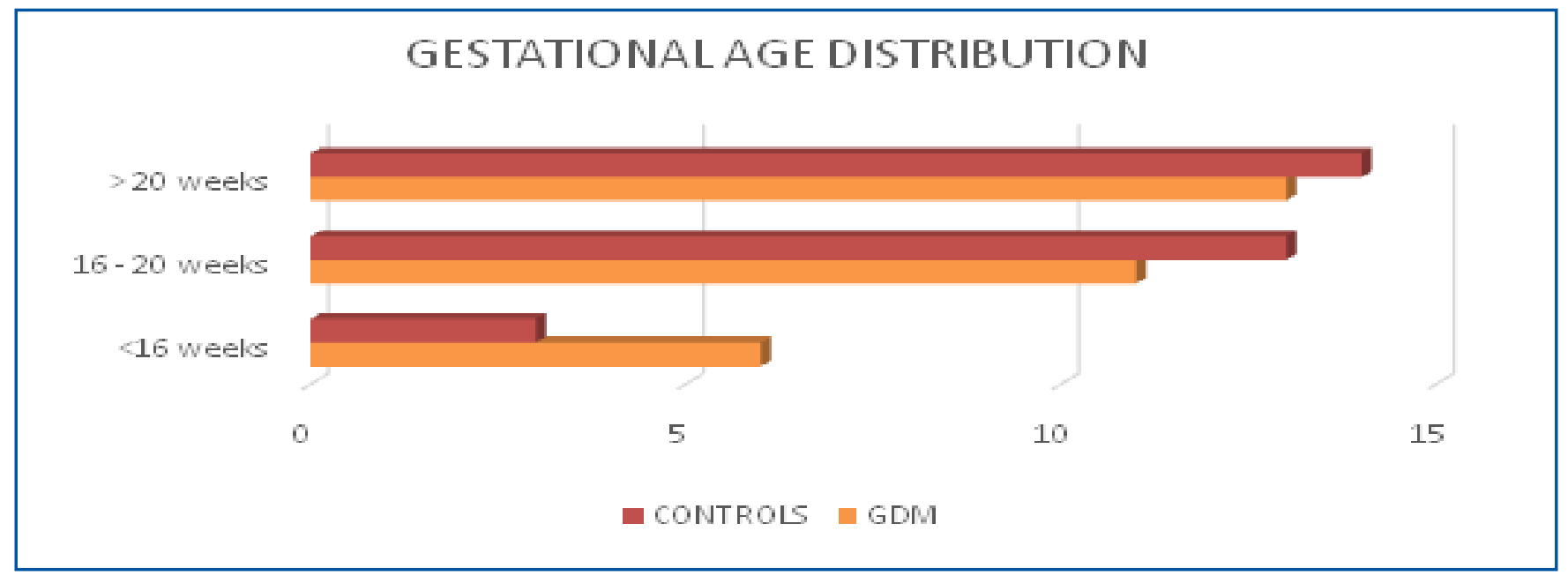

Fig. 2: Gestational age distribution among cases and controls.

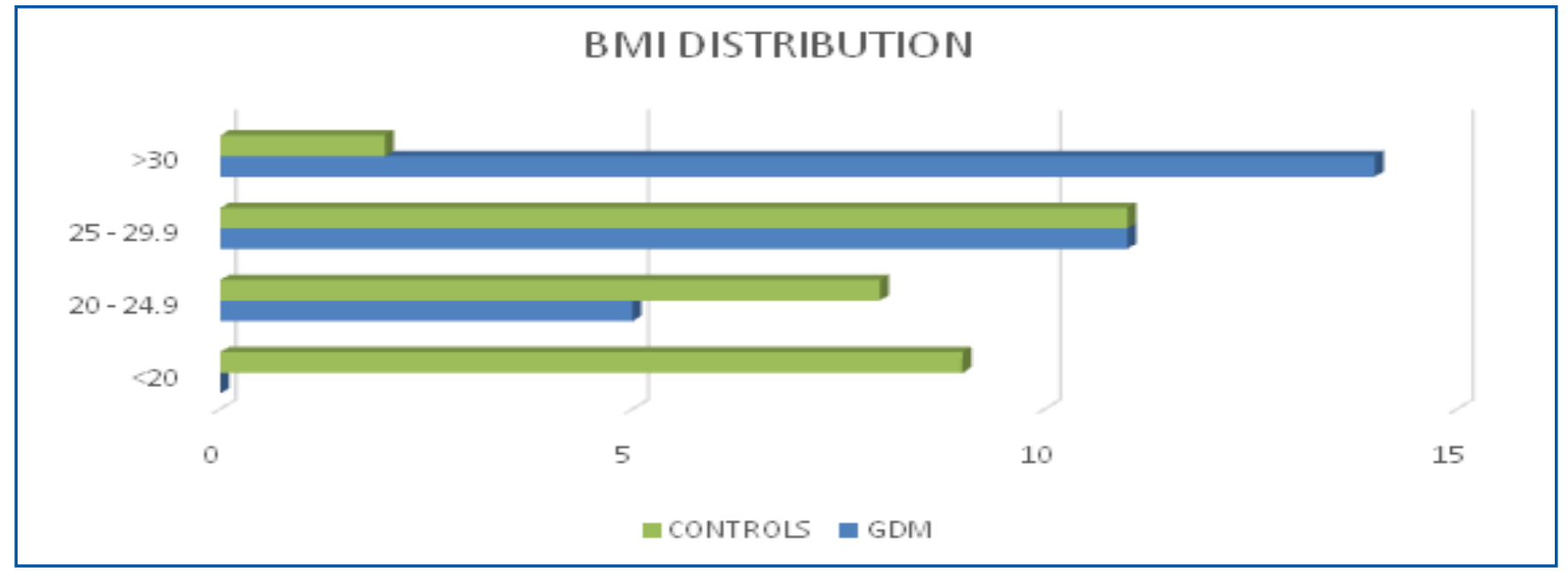

Fig. 3: BMI distribution among cases and controls. 


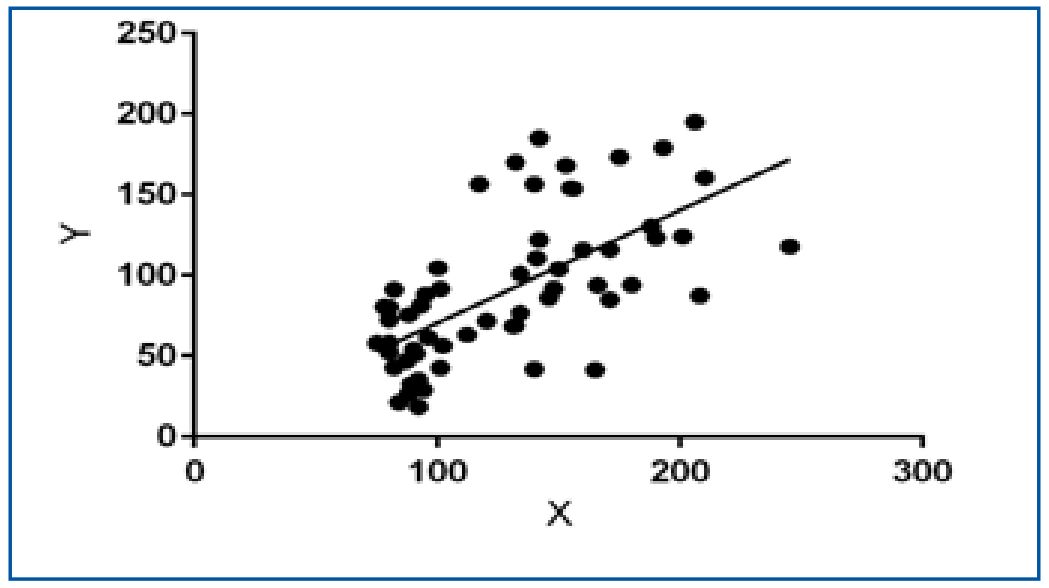

Fig. 4: Linear Regression analysis between LAP and OGCT.

\section{Discussion}

Pregnancy is invariably associated with progressive maternal hypertriglyceridemia and hypercholesterolemia. $(13,14)$ Occasionally a misadaptation occurs and these levels increase over a physiological range and dyslipidemia is recognized. It occurs in some pregnancies without causing any adverse pregnancy associated alterations and in some pregnancies coursing with pathologies as preeclampsia and gestational diabetes mellitus (GDM). This is called maternal supraphysiological hypercholesterolemia (MSPH).(15) This indicates that pregnancy induced hyperlipidemia contributes to an increase in the morbidity associated with GDM. (16)

The mean value of BMI and waist circumference among controls was $23.14 \pm 5.19$ and $82.49 \pm 6.67$ respectively and among cases was $28.81 \pm 4.11$ and $106.12 \pm 7.16$ respectively and these differences were statistically significant. ( $p$ value $<0.0001$ ) BMI and waist circumference were found to be significantly higher in cases than in controls, similar to findings by De Souza et al.(17)

The mean value of TGL among controls was $2.49 \pm 1.07$ and among cases was $1.84 \pm 0.69$ ( $p$ value $=0.002$ ). This exaggerated hypertriglyceridaemia is found throughout pregnancy, in a diabetic mother as demonstrated by Sanchez et al(15), Hollingsworth et al(18) and Kokkou et al(19) when compared to normal mothers. There are also studies where there was no significant change in plasma TGL levels among diabetic and non-diabetic pregnant women. (20)

The mean value of HDL among controls was $0.78 \pm$ 0.21 and among cases was $0.73 \pm 0.30$ ( $p$ value $=0.3499$ ). Though the difference in HDL levels was statistically insignificant, an increasing trend amongst the controls was observed which may be clinically significant. This lack of statistical significance can be substantiated based on the physiological drop in HDL levels in controls as shown in a population based study by Pusukuru et al. (21)

The mean LAP among controls was $62.89 \pm 30.68$ and among cases was $133.43 \pm 64.02$ and this difference was statistically significant ( $p$ value $<0.0001$ ) LAP values were significantly higher in cases compared to controls in parallel with the increase in TGL, BMI, waist circumference and decrease in HDL in cases.

Linear regression and correlation analysis between OGCT and LAP showed a strong positive correlation $(\mathrm{r}=0.6)$ which was also statistically significant $(\mathrm{p}<0.00001)$. Oh et al also showed a statistically significant correlation between OGCT and LAP, done in a study population of 2950 Korean women. (22)

Lipid accumulation product, based on simple anthropometry and lipid profile, can be used as a marker of metabolic disturbances in insulin resistance. In a population based cross sectional study, LAP has been shown to be better than BMI in identifying prevalent diabetes. (13) Brisson et al have shown that LAP is a useful risk marker of metabolic perturbations in young adult populations and that it could be used as a reliable marker among pregnant women also. (6)

\section{Conclusion}

The increased Lipid Accumulation Product (LAP) in GDM patients shows their elevated adipose tissue lipid levels; hence LAP can be used as a possible screening tool for GDM. Calculation of LAP in the first antenatal visit can be done to identify the degree of risk for developing GDM, as it has now been established that these indices are found to be elevated in diagnosed patients TGL levels were increased and HDL levels were decreased in our GDM patients when compared with controls, identification of which in the early trimester can reduce both maternal and foetal fatal outcomes. In future, the sample size can 
be increased and ethnic variations in maternal lipid levels should be studied to explore this association.

\section{Reference}

1. Expert Committee on the Diagnosis and Classification of Diabetes Mellitus: Report of the expert committee on the diagnosis and classification of diabetes mellitus. Diabetes Care 26 (Suppl.1):S5-S20, 2003

2. American Diabetes Association: Gestational diabetes mellitus (Position Statement). DiabetesCare 27 (Suppl. 1):S88-S90, 2004

3. Mokdad AH, Ford ES, Bowman BA, et al. Prevalence of obesity, diabetes, and obesityrelated health risk factors, 2001. JAMA. 2003;289(1):76-9.

4. Bower JF, Hadi H, Barakat HA. Plasma lipoprotein subpopulation distribution in Caucasian and AfricanAmerican women with gestational diabetes. Diabetes Care 2001;24:169-171

5. Gasevic D, Frohlich J, Mancini GBJ, Lear SA, "The association between triglyceride to high-density-lipoprotein cholesterol ratio and insulin resistance in a multiethnic primary prevention cohort," Metabolism: Clinical and Experimental, 2012;61,583-589.

6. Brisson D, Perron P, Guay SP, Gaudet D, Bouchard L. The "hypertriglyceridemic waist" phenotype and glucose intolerance in pregnancy. CMAJ 2010;182:E722-E725.

7. Lemieux I, Poirier P, Bergeron J, et al. Hypertriglyceridemic waist: a useful screening phenotype in preventive cardiology? Can J Cardiol 2007;23:23B-31B.

8. D.A.Enquobahrie,M.A.Williams, C.Qiu,andD.A.Luthy, "Early pregnancy lipid concentrations and the risk of gestational diabetes mellitus," Diabetes Research and Clinical Practice,vol. 70,no.2,pp.134-142,2005

9. Unger RH: Minireview: Weapons of Lean Body Mass Destruction: The Role of Ectopic Lipids in the Metabolic Syndrome. Endocrinology 2003, 144:5159-5165.

10. Schaffer JE: Lipotoxicity: when tissues overeat. Curr Opin Lipidol 2003, 14:281-287.

11. Unger RH: Lipid overload and overflow: metabolic trauma and the metabolic syndrome. Trends Endocrinol Metab 2003, 14:398-403

12. Linda A Barbour, MD, MSPH,Carrie E. Mccurdy, PHD,Teri L. Hernandez, RN, MS John P. Kirwan, PHD,Patrick M. Catalano, MD Jacob E. Friedman, PHD Cellular Mechanisms for Insulin Resistance in Normal Pregnancy and Gestational Diabetes Diabetes care:30(2), JULY 2007

13. Basaran A (2009) Pregnancy-induced hyperlipoproteinemia: review of the literature. Reproductive sciences 16:431-437.

14. Montes A, Walden CE, Knopp RH, Cheung M, Chapman MB, Albers JJ (1984) Physiologic and supraphysiologic increases in lipoprotein lipids and apoproteins in late pregnancy and postpartum. Possible markers for the diagnosis of "prelipemia". Arteriosclerosis 4:407-417.

15. Sanchez-Vera I, Bonet B, Viana M, Quintanar A, Martin MD, Blanco P, et al. (2007) Changes in plasma lipids and increased low-density lipoprotein susceptibility to oxidation in pregnancies complicated by gestational diabetes: consequences of obesity. Metabolism 56:1527-1533.

16. Kahn HS: The "lipid accumulation product" performs better than the body mass index for recognizing cardiovascular risk: a population based comparison. BMC Cardiovasc Disord 2005, 5:26.

17. De Souza LR, Kogan E, Berger H, et al. Abdominal adiposity and insulin resistance in early pregnancy.J 80 . Montelongo A, Lasuncion MA, Pallardo LF \& Herrera E. Longitudinal study of plasma lipoproteins and hormones during pregnancy in normal and diabetic women. Diabetes 1992; 41: 1651-1659.

18. Hollingsworth DR, Grundy SM. Pregnancy-associated hypertriglyceridemia in normal and diabetic women. Differences in insulin-dependent, non-insulindependent, and gestational diabetes. Diabetes. 1982;31(12):1092-7.

19. Koukkou E, Watts GF, Lowy C. Serum lipid, lipoprotein and apolipoprotein changes in gestational diabetes mellitus: a cross-sectional and prospective study. J Clin Pathol. 1996;49(8):634-7.

20. Rizzo M, Berneis $\mathrm{K}$, Altinova $\mathrm{AE}$ et al. Atherogenic lipoprotein phenotype and LDL size and subclasses in women with gestational diabetes. Diabet Med 2008; 25: 1406-1411.Obstet Gynaecol Can 2014;36:969-975

21. Raghuram Pusukur, Arjun S. Shenoi, Prakash KumarKyada, Babita Ghodke, Varshil Mehta, Kunal Bhuta, Aadhijaya Bhatia.Evaluation of Lipid Profile in Second and Third Trimester of Pregnancy.Journal of Clinical and Diagnostic Research. 2016 Mar, Vol-10(3): QC12-QC16

22. J.-Y. Oh, Y.-A. Sung and H. J. Lee. The lipid accumulation product as a useful index foridentifying abnormal glucose regulation in young Korean women. Diabet. Med. 30, 436-442 (2013).

*Corresponding author:

Dr. D. Padmalatha, Assistant Professor, Department of Obstetrics and Gynaecology, Government Kilpauk Medical College Hospital, Kilpauk, Chennai, Tamilnadu, INDIA

Phone: +91 9841696797

Email: endork@yahoo.com

Date of Submission : 05.04.2017

Date of Acceptance : 15.04.2017

Financial or other Competing Interests: None.

Date of Publication : 28.05.2017 